\title{
Cylindrical Permanent-Magnet Structures Using Images in an Iron Shield
}

\author{
Quanling Peng, S. M. McMurry, and J. M. D. Coey, Member, IEEE
}

\begin{abstract}
We report on cylindrical permanent-magnet structures that exploit the image effect in a surrounding circular soft-iron sheath. We present the theory for a general multipole ring, where the polarization direction $\alpha=(n+1) \psi, n$ is a positive or negative integer, and $\psi$ is the angular coordinate. For the uniformly magnetized case $n=-1$, a long cylindrical ring produces no field in its bore, and the field outside the cylinder is that of a linear dipole. When surrounded by the sheath, the field becomes uniform in the bore and zero outside the cylinder. Higher multipole fields can similarly be transformed from outward fields to inward fields by using the sheath. The field can be varied continuously by moving the sheath. We describe a small variable-field device using a Nd-Fe-B cylinder that produces a flux density of 0-400 mT.
\end{abstract}

Index Terms-Halbach cylinders, magnetic image effects, magnetic shielding, permanent-magnet flux sources.

\section{INTRODUCTION}

M ULTIPOLE magnetic fields can be generated using permanent-magnet rings [1]. Uniform dipolar fields are mostly used for physical measurements and materials processing, whereas quadrupole fields are useful for focusing charged-particle beams. The advantages of permanent magnets over electromagnets for these purposes are that the ring structures are comparatively light and compact and that they require no power supply or cooling [2]. Variable fields are available from structures of nested rotatable rings [3].

These ring structures became practicable only after the development of rare-earth permanent magnets with a sufficiently high anisotropy field for any element of the magnet assembly to be effectively transparent to the fields produced by the rest of the magnet. Halbach analyzed the ring designs [1] and showed that if an element with angular coordinate $\psi$ is magnetized at an angle $\alpha=(n+1) \psi$, then for $n= \pm N$, a pure $2 N$-pole magnet can be built. When $n=N$, the inward-field case, the field is confined in the bore of the ring, and it is zero outside; when $n=-N$, which is the outward-field case, the field is zero in the bore of the ring and nonzero outside. When $n=0$, there is no field, either inside or outside the ring. The rings are all supposed to be of infinite length along the $z$ direction in the cylindrical coordinate system. An illustration of these three cases is provided in Fig. 1. It is always possible to rotate the field pattern through

Manuscript received September 22, 2001; revised February 24, 2003. This work was supported by Enterprise Ireland under Contract ST 2000/025.

Q. Peng is with the Institute of High Energy Physics, Chinese Academy of Sciences, Beijing 100039, China (e-mail: pengql@ mail.ihep.ac.cn).

S. M. McMurry and J. M. D. Coey are with the Physics Department, Trinity

College, Dublin 2, Ireland (e-mail: smcmurry@tcd.ie; jcoey@tcd.ie).

Digital Object Identifier 10.1109/TMAG.2003.814286

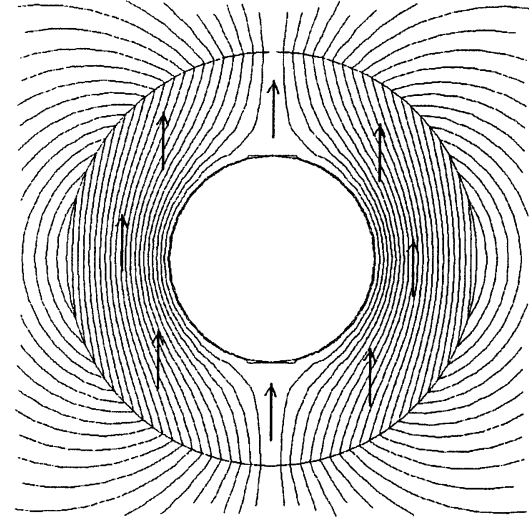

(a)

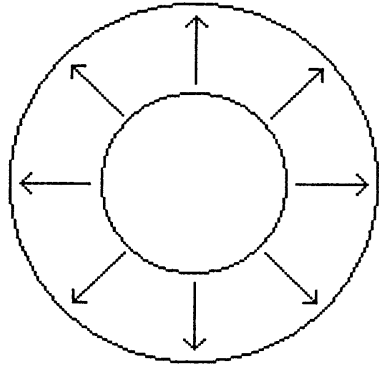

(b)

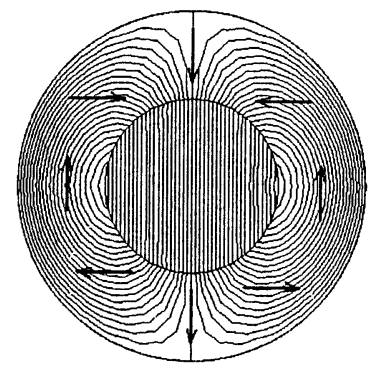

(c)
Fig. 1. Examples of infinitely long permanent-magnet ring structures generating pure multipole fields. (a) $n=-1, \alpha=0$. The outward-field ring, with uniform polarization, producing a dipole field outside the ring. (b) $n=0, \alpha=\psi$. The polarization is in the radial direction, and no field is produced either inside or outside the ring. (c) $n=+1, \alpha=2 \psi$. The inward-field dipole ring, producing a uniform field within the bore.

any angle $\beta$ by coherent rotation of the direction of polarization at each point in the ring. For example, when $\beta=\pi / 2$, the structure of Fig. 1(b) becomes a transversely magnetized toroid, which also produces no field anywhere.

In practice, it is difficult to construct magnets where the direction of polarization rotates continuously with position. Halbach [1] suggested making the rings from $M$ trapezoidal segments, where the easy directions in adjacent segments differ by $2(N+1) \pi / M$. The segmented construction introduces higher order multipoles into the field, and there may be errors in the orientation of the polarization and the size of the blocks that can be minimized by small radial displacements of the magnet segments [4], [5].

Finite cylinder length affects the field profile along the $z$ axis on a length scale comparable to the cylinder diameter. Both finite length and segmented construction introduce stray fields at the ends of the magnets which, for example, create torque 


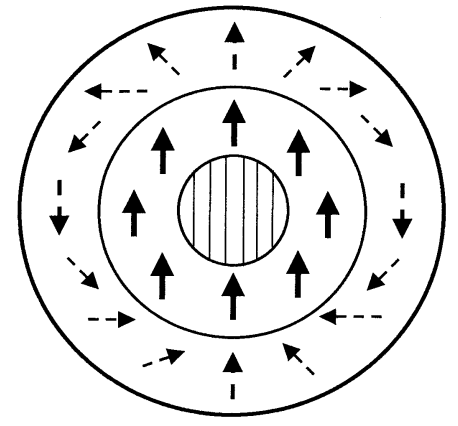

Fig. 2. Effect of a soft-iron sheath on the magnet of Fig. 1(a) is to produce an inward-field, like that of the magnet of Fig. 1(c).

in nested cylinders [6]. The fields produced at the ends of a finite-length magnet depend on $\beta$, when $n=0$.

It is possible to magnetize the cylinder in a multipole configuration with $n<0$ using an arrangement of external magnetizing coils [7], [8]. For example, an external dipole, quadrupole, or hexapole field pattern can be generated. Here, we show how it is possible to transform the external multipole field into one that is entirely confined in the bore of the magnet by using a soft iron sheath. These sheathed magnets are shielded from any external fields. The simplest example is a uniformly magnetized cylinder $n=-1$, which with a sheath produces a uniform field in the bore and no field outside the sheath as illustrated in Fig. 2.

All schemes for generating variable fields with a permanent magnet involve some sort of movement [2]. Switchable magnets involve movement of the magnet relative to a soft-iron section. Rotatable nested Halbach cylinders were proposed by Leupold [3], and the structures have been built and analyzed in detail [6]. They are used as laboratory variable flux sources, superseding the electromagnet, and fields of up to $2 \mathrm{~T}$ can be achieved. Arrangements of rotatable transversely magnetized rods [13] allow access to the field from three perpendicular directions. The maximum field obtainable is limited to $J / \mu_{0}$, and substantial torques are required to vary the field in structures with a small number of rods. In the present case, the magnet remains stationary, and the field is varied by lateral displacement of the sheath as indicated in Fig. 6.

Here, we first give the general theory of sheathed infinite length cylindrical multipole magnet structures using the idea of magnetic images in the surrounding iron. Then, we describe the construction and performance of a small dipole ring magnet. The field may be varied by moving the sheath along the $z$ axis rather than by moving the magnet.

\section{Cylindrical Magnetic Structures Shielded WITH SOFT IRON}

\section{A. Infinite Cylinders}

The permanent-magnet structures consist of a long hollow cylinder with inner and outer radii $R_{1}$ and $R_{2}$, respectively, surrounded by a soft iron sheath with inner radius $R_{3}$ (which is equal to $R_{2}$ in the absence of an air gap between magnet and sheath) and outer radius $R_{4}$. When the sheath is sufficiently thick for the iron to remain unsaturated, the fields in the regions $r<R_{3}$ may be calculated by an image method similar to that

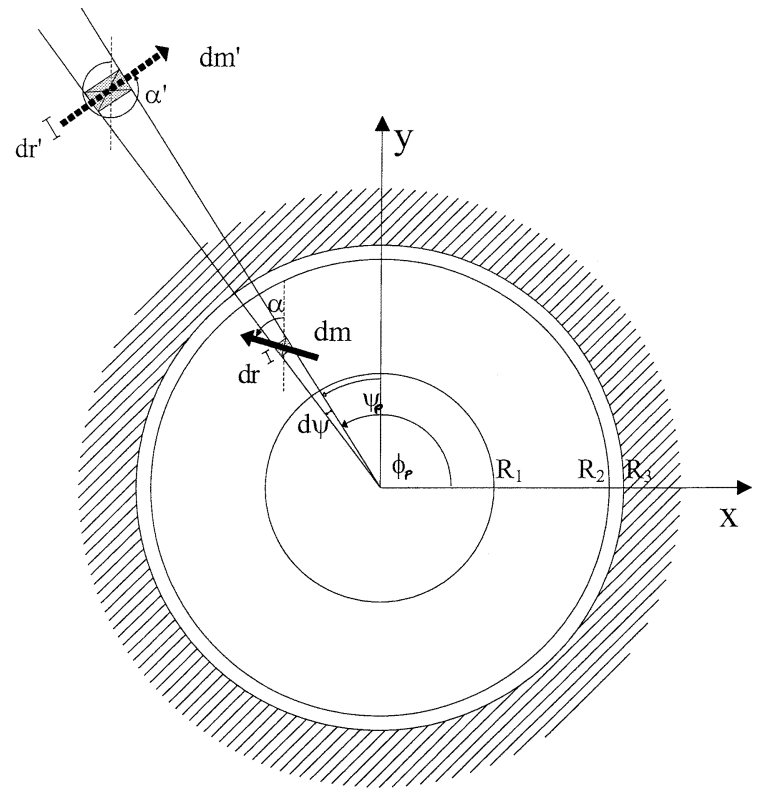

Fig. 3. The angular position of an element of polarization is defined by the angle $\psi=\phi-\pi / 2$, and the orientation of the dipole moment of the element $d \boldsymbol{m}$ is at angle $\alpha$ to the $y$ axis. The relative positions and orientations of a dipole element and its image in a cylindrical soft-iron shield are shown.

used in electrostatics. In the limit of infinite permeability, the soft iron corresponds to a perfect conductor and the magnetic field vanishes inside it. The $\boldsymbol{H}$ field is derived from the magnetic scalar potential $V(r, \phi, z)$, which satisfies Poisson's equation $\nabla^{2} V=-\rho_{m} / \mu_{o}$, with the polarization $\boldsymbol{J}$ of the permanent magnet providing the field source $\rho_{m}$. There is both a surface "charge" density $\boldsymbol{J} \cdot \hat{\boldsymbol{n}}$, where $\hat{\boldsymbol{n}}$ is the unit vector normal to the magnet surface, and a bulk "charge" density $-\nabla \cdot \boldsymbol{J}$. The boundary condition at the inner surface of the sheath is that the component of magnetic field parallel to the boundary vanishes

$$
H_{/ /}=-\hat{\boldsymbol{n}} \times\left.\nabla V\right|_{r=R_{3}}=0
$$

where $\hat{\boldsymbol{n}}$ is the unit outward normal to the boundary.

The coordinate system is shown in Fig. 3. A point $P$ in the ring has coordinates $\left(r_{P}, \psi_{P}\right)$, where $\psi_{P}$ is related to the conventional polar angle $\phi_{P}$ by $\psi_{P}=\phi_{P}-\pi / 2$. The magnetostatic potential in the region $r_{P}<R_{3}$ is of the form

$$
V=V_{o}+V^{\prime}
$$

where $V_{o}$ is the particular integral of the Poisson equation with a source term characteristic of the polarization in the ring $R_{1}<$ $r<R_{2}$. The additional potential $V^{\prime}$ represents the effect of the iron sheath, and satisfies the following conditions.

1) $V^{\prime}$ is a solution of the Laplace equation $\nabla^{2} V^{\prime}=0$ throughout the region $r_{P}<R_{3}$.

2) $V^{\prime}$ is chosen so that the total potential $V$ satisfies the boundary condition (1) at $r_{P}=R_{3}$.

3) $V^{\prime}$ must be regular at the origin.

When these conditions are satisfied, $V^{\prime}$ is the potential due to an image polarization in the region $r>R_{3}$.

Let the polarization $\boldsymbol{J}$ of the permanent magnet lie at an angle $\alpha(\psi)$ to the $y$ axis, which may vary with angular position $\psi$. Then, as shown in Appendix A, the image of an element of the 
permanent magnet at position $(r, \psi)$ is an element at $\left(r^{\prime}, \psi^{\prime}\right)$, where

$$
r^{\prime}=\frac{R_{3}^{2}}{r} \quad \psi^{\prime}=\psi
$$

Furthermore, the magnitude and orientation of the polarization in this image element are determined by

$$
J^{\prime}=\frac{r}{r^{\prime}} J \quad \alpha^{\prime}=2 \psi-\alpha .
$$

The first of (3) and (4) allow the magnetic moment in a small volume element of the image $d m^{\prime}=J^{\prime} r^{\prime} d r^{\prime} d \psi d z$ to be written in terms of that in the source as $d m^{\prime}=\left(R_{3} / r\right)^{2} d m$. The permanent magnet is assumed to be magnetically transparent, so that the field in the bore of the sheathed magnet is the sum of the fields produced there by all elements of the permanent magnet and their images. In the ideal case, there is no magnetic flux leakage to the region outside the soft iron sheath.

In the two-dimensional (infinite length) case, the multipole expansion of the field produced by a distribution of polarization is most easily obtained using a complex formalism [1]. As shown in Appendix B, polarization at $(r, \psi)$ contributes to the $2|n|$-pole component of the field only if its orientation satisfies

$$
\alpha=(n+1) \psi
$$

where $n$ is a positive or negative integer. [There is no field component in the expansion corresponding to $n=0$, as illustrated in Fig. 1(b).]

When the polarization in the permanent magnet at $\psi$ is oriented so that

$$
\alpha=(N+1) \psi, \quad N=1,2,3, \ldots
$$

the field is an inward one, being zero outside the ring and having a pure $2 N$-pole form inside it. For the case $N=1$, illustrated in Fig. 1(c), the field in the bore is (B10)

$$
\begin{aligned}
& H_{x}=0 \\
& H_{y}=\frac{J}{\mu_{o}} \ln \left(\frac{R_{2}}{R_{1}}\right) \quad r<R_{1} .
\end{aligned}
$$

For $N>1$, the field in the bore is (B9)

$$
\begin{gathered}
H_{x}=\frac{J}{\mu_{o}} \frac{N}{N-1}\left(\frac{1}{R_{1}^{N-1}}-\frac{1}{R_{2}^{N-1}}\right) r^{N-1} \sin (N-1) \psi \\
H_{y}=\frac{J}{\mu_{o}} \frac{N}{N-1}\left(\frac{1}{R_{1}^{N-1}}-\frac{1}{R_{2}^{N-1}}\right) r^{N-1} \cos (N-1) \psi \\
r<R_{1} .
\end{gathered}
$$

If, on the other hand, the orientation of the polarization at $\psi$ is

$$
\alpha=(-N+1) \psi
$$

the magnet produces an outward $2 N$-pole field, with

$$
\begin{gathered}
H_{x}=-\frac{J}{\mu_{o}} \frac{N}{N+1}\left(R_{2}^{N+1}-R_{1}^{N+1}\right) \frac{1}{r^{N+1}} \sin (N+1) \psi \\
H_{y}=+\frac{J}{\mu_{o}} \frac{N}{N+1}\left(R_{2}^{N+1}-R_{1}^{N+1}\right) \frac{1}{r^{N+1}} \cos (N+1) \psi \\
r>R_{2} .
\end{gathered}
$$

For $N=1$, Fig. 1(a), the ring is uniformly magnetized in the $y$ direction and produces a dipole field outside the ring.

The field produced by the image polarization is calculated similarly. Assuming $n=-N$, so that the permanent magnet produces an outward $2 \mathrm{~N}$-pole field, according to (4) and (5), the orientation of the image polarization at angular position $\psi$ is

$$
\alpha^{\prime}=(N+1) \psi
$$

The field within the bore is due entirely to the image polarization, which gives (B10)

$$
\begin{array}{r}
H_{x}^{\prime}=\frac{J}{\mu_{o}} \frac{N}{N+1} \frac{1}{R_{3}^{2 N}}\left(R_{2}^{N+1}-R_{1}^{N+1}\right) r^{N-1} \sin (N-1) \psi \\
H_{y}^{\prime}=\frac{J}{\mu_{o}} \frac{N}{N+1} \frac{1}{R_{3}^{2 N}}\left(R_{2}^{N+1}-R_{1}^{N+1}\right) r^{N-1} \cos (N-1) \psi \\
r<R_{1} . \quad(12)
\end{array}
$$

Notice that in the particular case $N=1$, the ring is uniformly magnetized in the $y$ direction, and as shown in Fig. 2, the image polarization is oriented in the same way as the permanent polarization for the case $n=+1$, illustrated in Fig. 1(c); the field in the bore is therefore uniform

$$
\begin{aligned}
& H_{x}=0 \\
& H_{y}=\frac{J}{2 \mu_{o}}\left(\frac{R_{2}^{2}-R_{1}^{2}}{R_{3}^{2}}\right), \quad r<R_{1} .
\end{aligned}
$$

This is in agreement with the result obtained by direct solution of the Poisson equation [9].

Equation (13) should be compared with the uniform inwardfield produced by the unshielded ring of Fig. 1(c) and (7). The dipole field produced by the shielded magnet (13) is always less than the inward field produced by a Halbach cylinder (7), and the flux density can never exceed $J / 2$. Nonetheless, the shielded configurations offer some benefits, which are discussed below.

The same is true for higher multipole fields. The table compares the flux density $\mu_{0} H$ in the bore of inward-field magnets obtained from (8) with that produced by sheathed outward-field magnets obtained from (12). Dimensions of the long cylinder are taken as $R_{1}=20 \mathrm{~mm}, R_{2}=R_{3}=40 \mathrm{~mm}$, and $J=$ $1000 \mathrm{mT}$.

\section{B. Finite Length Effects}

A real magnet cylinder is fairly short compared with its outer diameter, and for an outward-field magnet this results in a nonzero field within the bore. This field may be calculated using the surface charge model [10]. In the case of the outward-field dipole, the surface charge density is $+J \cos \psi$ on the surface $r=R_{2}$ and $-J \cos \psi$ on the surface $r=R_{1}$ and extends the length of the cylinder $-L<z<+L$. The field on the axis is $B_{y}(0,0, z)$

$$
\begin{aligned}
= & \frac{J}{4}\left\{(L+z)\left(\frac{1}{\sqrt{R_{1}^{2}+(L+z)^{2}}}-\frac{1}{\sqrt{R_{2}^{2}+(L+z)^{2}}}\right)\right. \\
& \left.+(L-z)\left(\frac{1}{\sqrt{R_{1}^{2}+(L-z)^{2}}}-\frac{1}{\sqrt{R_{2}^{2}+(L-z)^{2}}}\right)\right\} .
\end{aligned}
$$

Fig. 4(a) shows this field for three different lengths in the case $R_{1}=6 \mathrm{~mm}, R_{2}=20 \mathrm{~mm}$, and $J=1000 \mathrm{mT}$. As we expect, the field in the magnet approaches zero as the length $2 L$ becomes much greater than $R_{2}$. Just beyond the end of the magnet, the field direction is reversed. This reversal is a disadvantage in many applications. 

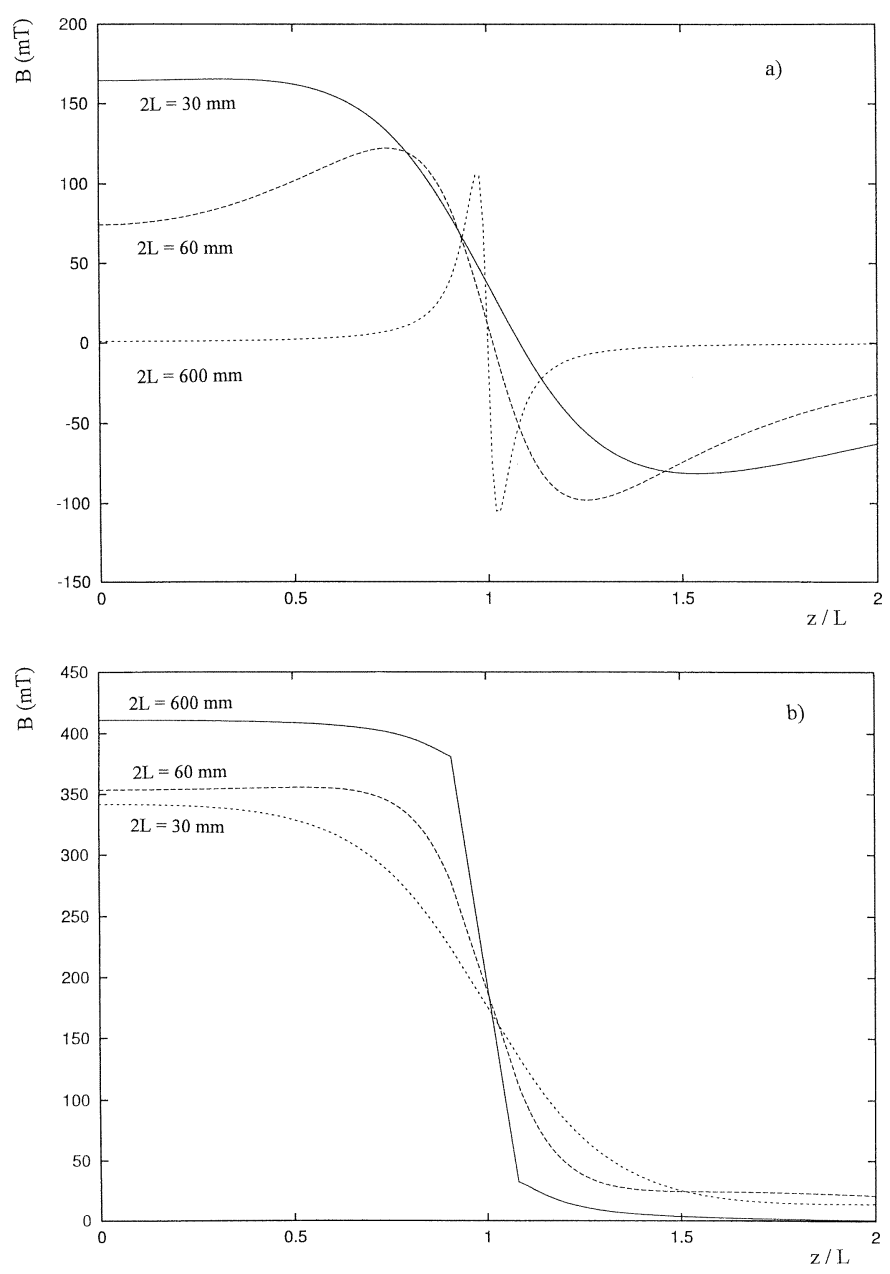

Fig. 4. Magnetic field produced on the axis of a uniformly magnetized ring with $J=1000 \mathrm{mT}, R_{1}=6 \mathrm{~mm}$, and $R_{2}=20 \mathrm{~mm}$ for lengths $l=2 L=$ 30,60 , and $600 \mathrm{~mm}$. (a) Without a sheath [calculated from (14)]. (b) With a sheath of inner radius $R_{3}=21 \mathrm{~mm}$ [calculated from (14) and (15)].

The effect of the finite length iron sheath may be estimated by replacing the soft iron by an outer permanent magnet with nonuniform magnetic charge density determined by (3) and (4). In this approximation, the field on the axis due to the sheath is

$$
\begin{aligned}
B_{y}^{\prime}(0,0, z) \\
=\frac{J}{4}\left\{\frac { 1 } { ( L + z ) } \left(\frac{R_{2}}{R_{3}^{2}} \sqrt{R_{3}^{4}+(L+z)^{2} R_{2}^{2}}\right.\right. \\
\left.-\frac{R_{1}}{R_{3}^{2}} \sqrt{R_{3}^{4}+(L+z)^{2} R_{1}^{2}}\right) \\
+\frac{R_{3}^{2}}{(L+z)^{2}} \ln \left(\frac{(L+z) R_{1}+\sqrt{R_{3}^{4}+(L+z)^{2} R_{1}^{2}}}{(L+z) R_{2}+\sqrt{R_{3}^{4}+(L+z)^{2} R_{2}^{2}}}\right) \\
+\frac{1}{(L-z)}\left(\frac{R_{2}}{R_{3}^{2}} \sqrt{R_{3}^{4}+(L-z)^{2} R_{2}^{2}}\right. \\
\left.-\frac{R_{1}}{R_{3}^{2}} \sqrt{R_{3}^{4}+(L-z)^{2} R_{1}^{2}}\right)+\frac{R_{3}^{2}}{(L-z)^{2}} \\
\left.\quad \times \ln \left(\frac{(L-z) R_{1}+\sqrt{R_{3}^{4}+(L-z)^{2} R_{1}^{2}}}{(L-z) R_{2}+\sqrt{R_{3}^{4}+(L-z)^{2} R_{2}^{2}}}\right)\right\}
\end{aligned}
$$

( $R_{3}$ is the inner radius of the sheath).

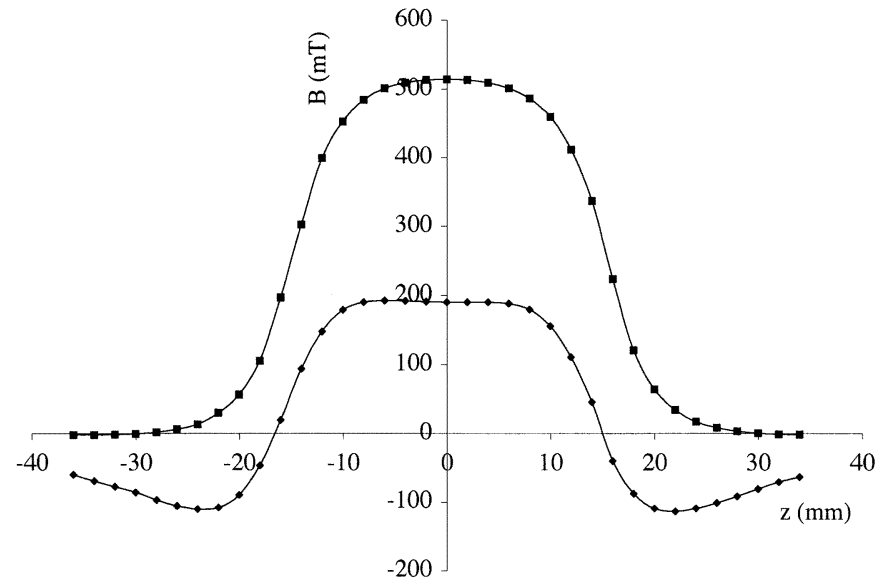

Fig. 5. Magnetic field produced on the axis of a short, uniformly magnetized ring, with (squares) and without (diamonds) a soft-iron sheath. The radial dimensions are those given in Fig. 4, and the length is $l=30 \mathrm{~mm}$.

The approximation to the total field on the axis of the sheathed magnet is the sum of the fields in (14) and (15). It is illustrated for three magnet lengths in Fig. 4(b). It is of particular interest that the effect of the sheath appears to counteract the field reversal beyond the end of the magnet. This effect was observed in measurements on the physical sheathed magnet described in Section III.

\section{PRactical Examples}

\section{A. Short Cylinder}

Fig. 5 shows the field produced along the axis of a short, uniformly magnetized $\mathrm{Nd}_{2} \mathrm{Fe}_{14} \mathrm{~B}$ ring $(n=-1)$ with dimensions $R_{1}=6, R_{2}=20, l(=2 L)=30$ (all in millimeters), and $J=1350 \mathrm{mT}$. The field in the bore at the center is $196 \mathrm{mT}$, compared with zero expected for a long cylinder. This illustrates the importance of end effects in short structures, where a large number of harmonics contribute significantly.

When the iron shield with $R_{3}=21 \mathrm{~mm}$ and $R_{4}=35 \mathrm{~mm}$ is in place, the central field rises to $515 \mathrm{mT}$, compared with $557 \mathrm{mT}$ predicted in the infinite-length limit by (13). Finite length effects are expected to reduce the magnitude of the field at the center. An interesting feature is that, while the field produced by the unsheathed magnet (and by the inward-field dipole ring) has the opposite sign outside the magnet to that in the center, this field reversal is eliminated in the sheathed magnet, as suggested by the approximate calculation in Section II-B.

\section{B. Cylinder With Moveable Sheath}

In order to create a magnetic field that is variable from zero to some maximum value $B_{\max }$, it is possible to exploit the end effect for finite cylinders. By leaving a small gap between two identical short dipole rings, the field at the center may be adjusted to zero or some negative value. The idea is illustrated by the lower curve in Fig. 7. The field can then be varied continuously by simultaneously advancing two half-sheaths to cover the permanent magnets as illustrated in Fig. 6. In our demonstrator magnets, two uniformly magnetized rings with $R_{1}=6, R_{2}=$ 20 , and $l=30 \mathrm{~mm}$ were separated by $4 \mathrm{~mm}$, and the soft-iron sheaths having $R_{3}=21 \mathrm{~mm}$ and $R_{4}=35 \mathrm{~mm}$ were moved 


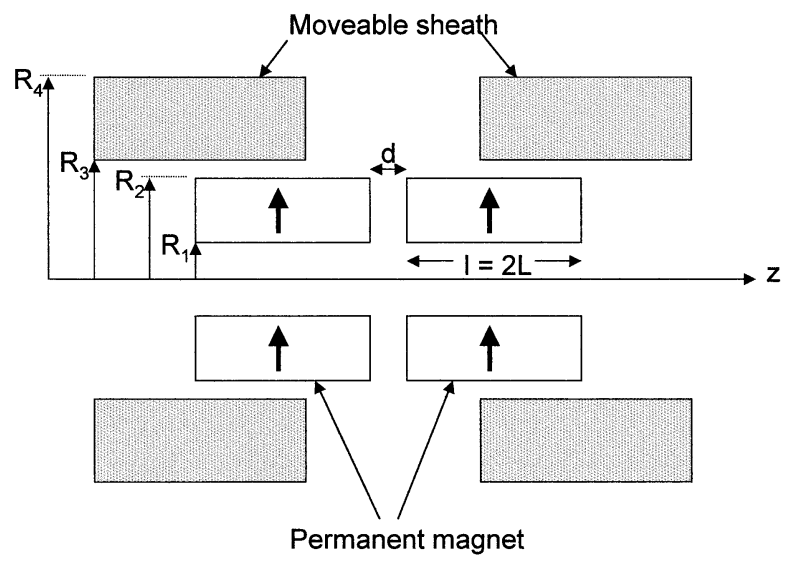

Fig. 6. Schematic arrangement for a variable flux source consisting of two permanent-magnet cylinders of length $l=2 L$ and inner and outer radii $R_{1}$ and $R_{2}$ separated by a small gap $d$, covered by two moveable soft iron sheaths of inner and outer radii $R_{3}$ and $R_{4}$

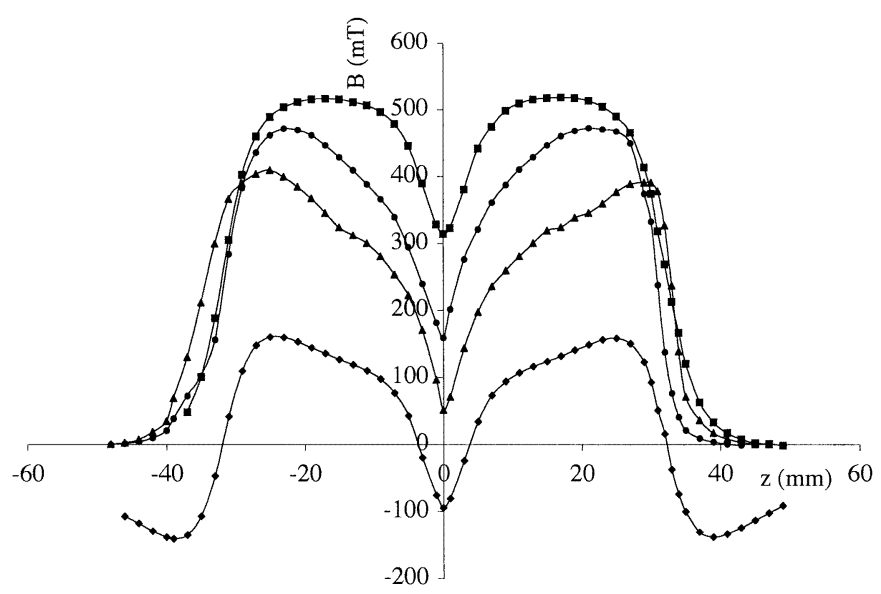

Fig. 7. Magnetic field produced on the axis of a magnet composed of two short uniformly magnetized rings separated by $4 \mathrm{~mm}$, showing the effect of the split soft-iron sheath illustrated in Fig. 6. The data show the field in the absence of the sheath (squares), with a central split in the sheath of width $4 \mathrm{~cm}$ (triangles) and $2.5 \mathrm{~cm}$ (diamonds), and with no separation between the two parts of the sheath (circles).

from either side by a system of screws. The curves in Fig. 7 show the field profile when the two halves of the sheath are separated by 40,25 , and $0 \mathrm{~mm}$. With this configuration, the field at the center varies from -95 to $310 \mathrm{mT}$. By choosing a gap of $1.4 \mathrm{~mm}$ between the rings, a variation from 0 to $410 \mathrm{mT}$ is achieved.

The shielded device was simulated with the help of software packages [11], [12].

\section{DISCUSSION AND CONCLUSION}

We have seen how it is possible to transform an outward $2 N$-pole field created by a permanent-magnet ring into an inward one by means of the image effect in a soft magnetic sheath, although the values obtained (Table I) are always less than those produced by an inward-field $2 N$-pole ring directly. So what are the benefits of the method? First, it is usually much simpler to create an outward-field $2 \mathrm{~N}$-pole magnet than an inward-field one. For $N=1$, it is easy to produce the uniformly magnetized hollow cylindrical rod of Fig. 1(a), but it is relatively dif-
TABLE I

Magnetic Field Produced IN THE Bore of Long INwARd Field AND SHIElded Outward FiEld $2 N$-Pole Cylinders, at RADIAL Position $r=10$, ASSUMING $R_{1}=20, R_{2}=R_{3}=40 \mathrm{~mm}$, AND $J=1000 \mathrm{mT}$

\begin{tabular}{|c|c|c|c|c|}
\hline $\mathbf{N}$ & Field & & $\begin{array}{c}\text { Inward-field, } \\
\text { mT }\end{array}$ & Sheathed outward-field, $\mathrm{mT}$ \\
\hline 1 & dipole & $\mu_{\mathrm{o}} \mathrm{H}$ & 693 & 375 \\
\hline 2 & quadrupole & $\mu_{0}|\mathrm{H}|_{r=10}$ & 500 & 146 \\
\hline 3 & hexapole & $\mu_{0}|\mathrm{H}|_{r=10}$ & 281 & 44 \\
\hline
\end{tabular}

ficult to make the dipole ring of Fig. 1(c). Following Halbach, the segmented design is usually employed, which requires cutting and assembly of numerous magnetized segments that are subject to strong forces. In the case of a quadrupole or hexapole field, an outward-field magnet can be created by magnetizing an isotropic, polycrystalline sintered, or bonded rare-earth or ferrite magnet in a pulsed field created by a system of external coils [7] and then adding the sheath to create an inward-field magnet. The magnitude of the field can be shimmed by varying the dimensions $R_{3}$ and $R_{4}$ of the soft-iron shield.

A second useful feature is the absence of a reverse dipole stray field along the axis. This allows samples to be brought into or out of the field of the magnet without seeing a stray field that might, for example, demagnetize them.

The third attractive feature of the shielded permanent magnets is their ability to generate continuously variable fields without moving the magnets and even change the sign of the field generated. Other schemes for generating variable fields with permanent magnets involve movement of the magnet. In the present case, the magnet remains stationary, and the field is varied by lateral displacement of the sheath.

In conclusion, we have shown how it is possible to manipulate the field in the bore of cylindrical permanent-magnet structures by means of a soft-iron sheath. These permanent-magnet structures may find applications for magnetic measurements, materials processing, and charged-particle beam control.

\section{APPENDIX A IMAGE METHOD}

Consider an infinite line dipole parallel to the axis within the bore of a thick infinite-length cylinder of inner radius $R_{3}$ made of perfectly permeable material, as shown in cross section in Fig. 3. The dipole is at $\boldsymbol{r}=(r, \psi)$ with $r<R_{3}$. (We are using the convention that angular position is measured from the $y$ axis). The general form for the potential at the point $P$ with coordinates $\left(r_{P}, \psi_{P}\right)$ due to a line dipole at $\boldsymbol{r}$ with moment per unit length $\lambda$ is

$$
V\left(r_{P}, \psi_{P}\right)=\frac{\lambda \cdot\left(\boldsymbol{r}_{P}-\boldsymbol{r}\right)}{2 \pi \mu_{o}\left(\boldsymbol{r}_{P}-\boldsymbol{r}\right)^{2}} .
$$

The $y$ axis may be defined to lie along the radius on which the dipole lies, so that the angular position of the dipole is $\psi=0$. If the dipole moment is at angle $\alpha$ to the $y$ axis, (A1) becomes

$$
\begin{aligned}
V\left(r_{P}, \psi_{P}\right) & =\frac{\lambda}{2 \pi \mu_{o}} \\
\times & \frac{\left\{\left(r_{P} \cos \psi_{P}-r\right) \cos \alpha+r_{P} \sin \psi_{P} \sin \alpha\right\}}{r_{P}^{2}-2 r_{P} r \cos \psi_{P}+r^{2}}
\end{aligned}
$$


and the component of the magnetic field parallel to the surface is

$$
\begin{aligned}
& H_{\psi}\left(r_{P}, \psi_{P}\right)=-\frac{1}{r_{P}} \frac{\partial V}{\partial \psi_{P}}=-\frac{\lambda}{2 \pi \mu_{o}} \\
& \cdot \frac{\left\{\left(\left(r_{P}^{2}+r^{2}\right) \cos \psi_{P}-2 r_{P} r\right) \sin \alpha-\left(r_{P}^{2}-r^{2}\right) \sin \psi_{P} \cos \alpha\right\}}{\left(r_{P}^{2}-2 r_{P} r \cos \psi_{P}+r^{2}\right)^{2}} .
\end{aligned}
$$

At the surface $r_{P}=R_{3}$, the contribution of this field component must be cancelled by the $\psi_{P}$ component of an additional field $\boldsymbol{H}^{\prime}$ derived from a potential $V^{\prime}$ satisfying conditions 1 ), 2 ), and 3) of Section II-A in the region $r_{P}<R_{3}$. Clearly, at $r_{P}=R_{3}$, the field component $H_{\psi}^{\prime}$ must have the same $\psi_{P}$ dependence as $H_{\psi}$ in order to satisfy the boundary condition (1) for all $\psi_{P}$. Consequently, it is reasonable to assume that $V^{\prime}$ is a dipole potential, but in order for it to satisfy the Laplace equation for all $r_{P}<R_{3}$, its source must lie outside this region.

Assuming that $V^{\prime}$ is due to a line dipole of moment $\lambda^{\prime}$ at position $\boldsymbol{r}^{\prime}=\left(r^{\prime}, \psi^{\prime}\right)$, we determine the values of these parameters for which $H_{\psi}^{\prime}$ cancels the field $H_{\psi}$ due to the real dipole at the boundary. For reasons of symmetry, the image dipole lies on the same radius as the real dipole; consequently, $\psi^{\prime}=\psi=0$. The potential due to this dipole is of exactly the same form as $V$ (A2), with $\lambda^{\prime}, r^{\prime}$, and $\alpha^{\prime}$ replacing $\lambda, r$, and $\alpha$. Similarly, these substitutions in (A3) give the component of the magnetic field parallel to the boundary due to the image dipole $H_{\psi}^{\prime}$. The condition for this field to cancel the corresponding component of the real dipole field at the boundary $r_{P}=R_{3}$ is

$$
H_{\psi}^{\prime}\left(R_{3}, \psi_{P}\right)=-H_{\psi}\left(R_{3}, \psi_{P}\right)
$$

for all $\psi_{P}$. This may be written

$$
\begin{aligned}
& \lambda^{\prime} \frac{2 R_{3} r^{\prime} \sin \alpha^{\prime}}{\left(R_{3}^{2}-2 R_{3} r^{\prime} \cos \psi_{P}+r^{\prime 2}\right)^{2}} \\
& \times\left\{1-\frac{\left(R_{3}^{2}+r^{\prime 2}\right)}{2 R_{3} r^{\prime}} \cos \psi_{P}+\frac{\left(R_{3}^{2}-r^{\prime 2}\right) \cot \alpha^{\prime}}{2 R_{3} r^{\prime}} \sin \psi_{P}\right\} \\
&=-\lambda \frac{2 R_{3} r \sin \alpha}{\left(R_{3}^{2}-2 R_{3} r \cos \psi_{P}+r^{2}\right)^{2}} \\
& \quad \times\left\{1-\frac{\left(R_{3}^{2}+r^{2}\right)}{2 R_{3} r} \cos \psi_{P}\right. \\
&\left.\quad+\frac{\left(R_{3}^{2}-r^{2}\right) \cot \alpha}{2 R_{3} r} \sin \psi_{P}\right\} .
\end{aligned}
$$

In order for this to be satisfied for all $\psi_{P}$, the coefficients of $\cos \psi_{P}$ within the braces on either side of the equation must be equal, as must the coefficients of $\sin \psi_{P}$ and the factors outside the braces. These requirements are met by

$$
r^{\prime}=\frac{R_{3}^{2}}{r}, \quad \frac{\lambda^{\prime}}{\lambda}=\frac{r^{\prime}}{r}, \quad \alpha^{\prime}=-\alpha .
$$

The final equality indicates that the component of the image dipole parallel to the surface is reversed relative to the real dipole.

For a dipole at the general position $(r, \psi)$, the image dipole is at $\left(r^{\prime}, \psi^{\prime}\right)$, where $r^{\prime}$ is given in (A6), and

$$
\psi^{\prime}=\psi \text {. }
$$

The moment of the real dipole is at angle $\alpha-\psi$ to the radial direction, as shown in Fig. 3, and the reversal of the transverse component of the image dipole requires $\alpha^{\prime}-\psi=-(\alpha-\psi)$, so that

$$
\alpha^{\prime}=2 \psi-\alpha .
$$

If the dipole is due to a volume element of polarization density $J$, its moment is

$$
d \lambda=J r d r d \psi[\cos (N \psi) \hat{\boldsymbol{r}}-\sin (N \psi) \hat{\psi}]
$$

and the image dipole moment is

$$
d \lambda^{\prime}=J^{\prime} r^{\prime} d r^{\prime} d \psi^{\prime}\left[\cos \left(N \psi^{\prime}\right) \hat{\boldsymbol{r}}+\sin \left(N \psi^{\prime}\right) \hat{\psi}\right] .
$$

Since $\left(r^{\prime}, \psi^{\prime}\right)$ is related to $(r, \psi)$ through (A6) and (A7), while the image element subtends the same angle $d \psi$ as the real element, the volume element of the image is larger by a factor $\left(r^{\prime} / r\right)^{2}$. However, according to (A6), $d \lambda^{\prime}$ is a factor $r^{\prime} / r$ greater than $d \lambda$. Thus, the polarization density $J^{\prime}$ is smaller than $J$ by a factor $r / r^{\prime}=\left(R_{3} / r^{\prime}\right)^{2}$ as seen in (4).

The image potential $V^{\prime}$ satisfies the requirements 1), 2), and 3) of Section II-A, and it is unique, since a solution of the Laplace equation satisfying particular boundary conditions is unique. Thus, the effect of the sheath may be represented by the presence of an image polarization with the parameters given in (3) and (4).

\section{APPENDIX B}

\section{CALCUlation of the Field Using Complex Notation}

The magnetostatic field in two dimensions is an analytic function of the complex variable [1]

$$
z=x+i y=r e^{i \phi}=r e^{i(\psi+\pi / 2)}
$$

and the multipole expansion of the field is

$$
H^{*}(z)=H_{x}-i H_{y}=\sum_{n} b_{n} z^{n-1} .
$$

The summation is over all positive integers $n$ for a bounded region including the origin (such as $r<R_{1}$ ) and over all negative integers $n$ for an unbounded region excluding the origin (such as $\left.r>R_{2}\right)$. The coefficients in this expansion are

$$
b_{n}=\frac{|n|}{2 \pi \mu_{o}} \iint r^{\prime} d r^{\prime} d \psi^{\prime} \frac{J\left(z^{\prime}\right)}{z^{\prime n+1}} .
$$

Let the polarization in the ring be

$$
\boldsymbol{J}(\psi)=J(-\sin \alpha(\psi) \hat{\boldsymbol{x}}+\cos \alpha(\psi) \hat{\boldsymbol{y}}) .
$$

This may be rewritten as a complex function

$$
J=J_{x}+i J_{y}=J e^{i(\alpha(\psi)+\pi / 2)} .
$$

Then, (B3) gives

$$
\begin{aligned}
b_{n}=\frac{|n|}{2 \pi \mu_{o}} \int_{R_{1}}^{R_{2}} r^{\prime} d r^{\prime} & \frac{J}{r^{\prime(n+1)}} \int_{0}^{2 \pi} d \psi^{\prime} \\
& \times e^{i\left(\alpha\left(\psi^{\prime}\right)+\pi / 2\right)} e^{-i(n+1)\left(\psi^{\prime}+\pi / 2\right)} .
\end{aligned}
$$

The integral over $\psi^{\prime}$ vanishes unless

$$
\alpha=(n+1) \psi^{\prime} .
$$


In the case of uniform polarization, as in the permanent magnet, the integral over $r^{\prime}$ gives

$$
b_{n}=\frac{J}{\mu_{o}} \begin{cases}e^{-i n \pi / 2} \frac{|n|}{(-n+1)}\left(R_{2}^{-n+1}-R_{1}^{-n+1}\right), & n \neq 1 \\ -i \ln \frac{R_{2}}{R_{1}}, & n=1 .\end{cases}
$$

This equation determines the expansion coefficients for the field (B2) in the region within the ring $r<R_{1}$ for $n>0$, and in the region $r>R_{2}$ for $n<0$. The $2|n|$-pole component of the field is

$$
\begin{aligned}
H_{x}-i H_{y}= & \frac{J}{\mu_{o}} \frac{|n|}{(-n+1)}\left(R_{2}^{-n+1}-R_{1}^{-n+1}\right) r^{n-1} \\
& \times(\sin (n-1) \psi-i \cos (n-1) \psi), \quad n \neq+1
\end{aligned}
$$

$$
H_{x}-i H_{y}=-i \frac{J}{\mu_{o}} \ln \left(\frac{R_{2}}{R_{1}}\right), \quad n=+1
$$

In the case $n>0$, the field must vanish in the region $r>R_{2}$; otherwise, it would diverge as $r \rightarrow \infty$. Similarly, for $n<0$, the field must vanish in the region $r<R_{1}$ in order to remain regular at the origin.

In the case $n=-N$, the image polarization density satisfies (4), giving $\alpha^{\prime}=(N+1) \psi^{\prime}$. The field in the core due to the image is determined by the coefficient

$$
\begin{aligned}
b_{N}^{\prime} & =\frac{N}{\mu_{o}} \int_{R_{3}^{2} / R_{2}}^{R_{3}^{2} / R_{1}} r^{\prime} d r^{\prime} \frac{J^{\prime}}{r^{\prime N+1}} e^{-i N \pi / 2} \\
& =\frac{J}{\mu_{o}} \frac{N}{N+1} \frac{e^{-i N \pi / 2}}{R_{3}^{2 N}}\left(R_{2}^{N+1}-R_{1}^{N+1}\right) .
\end{aligned}
$$

The corresponding magnetic field is

$$
\begin{aligned}
H_{x}^{\prime}-i H_{y}^{\prime}=\frac{J}{\mu_{o}} & \frac{N}{N+1}\left(\frac{R_{2}^{N+1}-R_{1}^{N+1}}{R_{3}^{2 N}}\right) r^{N-1} \\
& \times(\sin (N-1) \psi-i \cos (N-1) \psi) .
\end{aligned}
$$

\section{APPENDIX C \\ ERROR EFFECTS}

Magnetic field errors mainly come from: 1) nonuniformity of the polarization $\Delta J ; 2)$ errors in the easy axis direction $\Delta \alpha$, which for a dipole is the same as the permanent ring rotation about the center by $\Delta \alpha ; 3$ ) errors in the permanent cylinder radius $\Delta R_{i}, i=1,2$; and 4 ) errors in the iron shield radius $\Delta R_{3}$. In each case, the first-order error can easily be obtained by differentiating (B6), and in the case $n=-N$, one obtains

$$
\begin{aligned}
\Delta b_{N} & =\frac{\Delta J}{J} b_{N} \\
\Delta b_{N} & =i \Delta \alpha b_{N} \\
\Delta b_{N} & =(N+1) \frac{\Delta R_{i}}{R_{i}} b_{N}, \quad i=1,2 \\
\Delta b_{N} & =-2 N \frac{\Delta R_{3}}{R_{3}} b_{N} .
\end{aligned}
$$

\section{ACKNOWLEDGMENT}

The authors wish to thank M. O'Reilly for the mechanical work and R. Gunning for help with the diagrams.

\section{REFERENCES}

[1] K. Halbach, "Design of permanent multipole magnets with oriented rareearth cobalt material," Nucl. Instr. Methods, vol. 169, pp. 1-10, 1980.

[2] R. Skomski and J. M. D. Coey, Permanent Magnetism. Bristol, U.K.: IOP, 1999.

[3] H. Leupold, Rare-Earth Iron Permanent Magnets, J. M. D. Coey, Ed. Oxford, U.K.: Clarendon, 1996, ch. 8.

[4] K. Halbach, "Perturbation effects in segmented rare earth cobalt multipole magnets," Nucl. Instr. Methods, vol. 198, pp. 213-215, 1982.

[5] Q. L. Peng et al., "Construction and tuning of BEPC mini- $\beta$ permanent quadrupole prototype," Nucl. Instr. Methods A, vol. 406, pp. 53-57, 1998.

[6] T. R. Ni Mhiochain, D. Weaire, S. M. McMurry, and J. M. D. Coey, "Analysis of torque in nested magnetic cylinders," J. Appl. Phys., vol. 86, pp. 6412-6424, 1999.

[7] G. W. Jewell, D. Howe, and C. D. Riley, "The design of radial-field multipole impulse magnetizing fixtures for isotropic NdFeB magnets," IEEE Trans. Magn., vol. 33, pp. 708-722, Jan. 1997.

[8] Z. Q. Zhu et al., "Analysis of anisotropic bonded NdFeB Halbach cylinders accounting for partial powder alignment," IEEE Trans. Magn., vol. 36, pp. 3575-3577, Sept. 2000.

[9] M. G. Abele, Structures of Permanent Magnets. New York: Wiley, 1993, ch. 2.2.

[10] J.-P. Yonnet, Rare-Earth Iron Permanent Magnets, J. M. D. Coey, Ed. Oxford, U.K.: Clarendon, 1996, ch. 9.

[11] Reference Manual for the Poisson/Superfish Group of Codes, 1987. LA-UR-87-126.

[12] Reference Manual for the MAGNET 6.6, Infolytica Corporation, Montreal, QC, Canada.

[13] O. Cugat, P. Hansson, and J. M. D. Coey, "Permanent magnet variable flux sources," IEEE Trans. Magn., vol. 30, pp. 4602-4604, Nov. 1994. 